ESAIM: PROCEEDINGS, February 2007, Vol.16, 77-88

Eric Cancès \& Jean-Frédéric Gerbeau, Editors

DOI: $10.1051 /$ proc:2007014

\title{
TOWARDS IMPLICIT SUBGRID-SCALE MODELING BY PARTICLE METHODS
}

\author{
S. Hickel ${ }^{1}$, L. Weynans ${ }^{2}$, N.A. Adams ${ }^{3}$ And G.-H. Cottet ${ }^{4}$
}

\begin{abstract}
The numerical truncation error of vortex-in-cell methods is analyzed a-posteriori through the effective spectral numerical viscosity for simulations of three-dimensional isotropic turbulence. The interpolation kernels used for velocity-smoothing and re-meshing are identified as the most relevant components affecting the shape of the spectral numerical viscosity as a function of wave number. A linear combination of well-known standard kernels leads to new kernels assigned to the specific use for implicit large-eddy simulation of turbulent flows, i.e. their truncation errors acts as subgrid-scale model. Numerical results are provided to show the potential and drawbacks of the approach.
\end{abstract}

\section{INTRODUCTION}

In Large-Eddy Simulation (LES) of turbulent flows the evolution of non-universal larger scales is computed, whereas their interaction with unresolved subgrid scales (SGS) is modeled. Modeling SGS effects amounts to the modification of the underlying conservation laws to remove the coupling with spatial and temporal scales which cannot be resolved by the numerical grid functions. A mathematical framework based on explicit filtering was proposed by Leonard [18]. Following his concept, explicit SGS models were commonly derived without reference to a computational grid and without taking into account a discretization scheme. It should be noted that Leonard's ansatz implies a subsequent discretization of the filtered equations. However, numerically computed SGS stresses are affected by the truncation error of the discretization scheme [11]. This interference between subgrid-scale model and numerical discretization can result in an unpredictable lack of accuracy of the LES. Along with the change of turbulence structure near flow boundaries, such as walls or fluid interfaces, it constitutes a major obstacle for the application of LES for standard use in industrial research and development.

SGS effects are modeled explicitly if the underlying conservation law is modified and subsequently discretized. Employing an explicit SGS model, Schumann [22] already argued that discretization effects should be taken into account within the SGS model formulation. Systematic investigation of the problem $[11,17]$ showed that within the standard formulation of eddy-viscosity SGS models a second-order accurate numerical discretization is insufficient in general.

As an alternative to standard formulations, Implicit Large-Eddy Simulation (ILES) denotes the situation when the unmodified conservation law is discretized and the numerical truncation error acts as SGS model. Since the SGS model is implicitly contained within the discretization scheme an explicit computation of model terms becomes unnecessary. Implicit SGS modeling requires systematic procedures for design and analysis of appropriate discretization schemes. A connection between grid-truncation and the filtering approach can be

\footnotetext{
${ }^{1}$ Institute of Aerodynamics, Technische Universität München, D-85747 Garching, Germany. E-mail: sh@tum.de

2 CEA-DIF, BP 1291680 Bruyéres-le-Châtel, France.

3 Institute of Aerodynamics, Technische Universität München, D-85747 Garching, Germany.

${ }^{4}$ LMC-IMAG, Université Joseph Fourier, BP 53 Grenoble Cédex 9 , France.
} 
made through numerical methods which employ an inherent filter operation. There are two numerical methods with inherent filtering: the finite-volume method and the particle method.

One recent approach employing a finite-volume discretization is the Adaptive Local Deconvolution Method (ALDM) [1,14]. ALDM was developed using a systematic framework for design, analysis, and optimization of nonlinear discretization schemes for implicit LES. In this framework parameters inherent to the discretization scheme are determined in such a way that the numerical truncation error acts as a physically motivated SGS model. Optimal model parameters for ALDM were determined by systematically minimizing a cost function which measures the difference between spectral numerical viscosity of the discretization and the eddy viscosity from EDQNM theory for isotropic turbulence. With the optimized discretization parameters ALDM matches the theoretical requirements of EDQNM. The spectral eddy viscosity of the ALDM scheme exhibits a lowwavenumber plateau at the correct level and reproduces the typical cusp shape up to the cut-off wave number at the correct magnitude, see figure 1b. Computational results for LES with ALDM show that an implicit model can perform at least as well as established explicit models [14-16]. For a comprehensive review on alternative approaches to ILES, see the textbook of Grinstein et al. [12].

The present paper is dedicated to the extension of the implicit modeling methodology to particle methods for the LES of incompressible turbulent flows. Particle methods are Lagrangian techniques that have been proposed as an alternative to more conventional grid-based methods. Their conceptional advantages over purely gridbased methods are self-adaptivity and numerical stability. Due to the Lagrangian formulation, the inertial non-linear term in the flow equations is implicitly accounted for by the transport of particles such that the resulting method is not subject to the convective stability constraint.

Being a Lagrangian method, the convection of particles requires the reconstruction of the velocity field. The choice of Particle-In-Cell (PIC) methods, rather than totally grid-free particle methods, is dictated by the fact that for three-dimensional incompressible flows particle methods are in general based on the vorticity formulations of the Navier-Stokes equations. The velocity calculation is much less expensive when it relies on grid-based Poisson solver than when it uses N-body type summation formulas. In the context of LES the PIC approach inherently contains a kernel-regularization for reconstructing the velocity field from the vorticity field. The discretization particles are convected with this smoothed velocity field, thus providing a regularized solution [7]. Cottet [6] has demonstrated that a regularized kernel amounts to a modified transport equation whose structure resembles the Navier-Stokes- $\alpha$ model of Domaradzki and Holm [9]. Additional contributions to modeling of non-resolved scales arise from a re-meshing procedure required to maintain consistency of the discretization. Analyzing the modified differential equation Cottet and Weynans [8] found that re-meshing once each time step results in an effective discretization which is equivalent to well-known finite-difference schemes for the Eulerian formulation.

An approach to the optimization of these two contributions with respect to implicit SGS modeling is detailed in the following.

\section{THE VORTEX-IN-CELL METHOD}

We consider the evolution of a vorticity field in a three dimensional incompressible viscous flow. The NavierStokes equations in velocity-vorticity formulation read

$$
\begin{gathered}
\frac{\partial \boldsymbol{\omega}}{\partial t}+\boldsymbol{u} \cdot \boldsymbol{\nabla} \boldsymbol{\omega}-\boldsymbol{\omega} \cdot \boldsymbol{\nabla} \boldsymbol{u}-\nu \boldsymbol{\nabla} \cdot \boldsymbol{\nabla} \boldsymbol{\omega}=\mathbf{0}, \\
\boldsymbol{u}=\boldsymbol{\nabla} \times \boldsymbol{\psi}, \\
\boldsymbol{\nabla} \cdot \boldsymbol{\nabla} \boldsymbol{\psi}=-\boldsymbol{\omega},
\end{gathered}
$$

where $\boldsymbol{\omega}$ is the vorticity, $\boldsymbol{\psi}$ is the vector potential, $\boldsymbol{u}$ is the velocity, and $\nu$ is the kinematic viscosity. This system has to be supplemented with appropriate initial and boundary conditions. Throughout the present paper the computational domain is a triply-periodic box with periodicity length $L=2 \pi$. 
Vortex-in-Cell (VIC) methods are based on the Lagrangian formulation of these equations, which has the advantage of a robust and accurate treatment of the convection terms in eq. (1a). That is, only vortex stretching

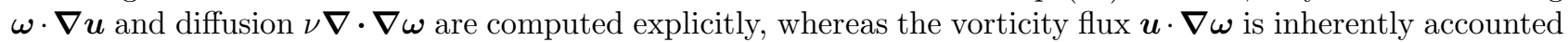
for by the advection of particles

$$
\frac{\partial \boldsymbol{\omega}}{\partial t}+\boldsymbol{u} \cdot \nabla \boldsymbol{\omega}=\frac{D \boldsymbol{\omega}}{D t}
$$

The Poisson equations (1c) are solved on an Eulerian grid. Periodic boundary conditions allow to employ fast FFT-based Poisson solvers.

For an inviscid flow, Helmholtz's and Kelvin's theorems assert the conservation of the circulation of material elements. More specifically, for VIC methods the idea is to use particles to transport approximately conserved quantities (circulation) and grid based formulas to compute auxiliary fields (velocity and strain). Transfers between grid and particles are carried out with interpolation formulas, which will be described in the following section. The computational domain is sampled into cells with volume $v_{p}$ for which the circulation $\int \boldsymbol{\omega}(., t) d \boldsymbol{x}$ is represented by a single particle. Thus the particle approximation of the vorticity field is

$$
\boldsymbol{\omega}(x)=\sum_{p} \boldsymbol{\alpha}_{p} \delta\left(\boldsymbol{x}-\boldsymbol{x}_{p}\right)=\sum_{p} v_{p} \boldsymbol{\omega}_{p} \delta\left(\boldsymbol{x}-\boldsymbol{x}_{p}\right) .
$$

where $\boldsymbol{x}_{p}, \boldsymbol{\alpha}_{p}, \boldsymbol{\omega}_{p}$ and $v_{p}$ denote the location, circulation, vorticity and volume of the respective particle.

The Lagrangian formulation of particle methods avoids the explicit discretization of the convective term in the governing transport equations and the associated stability constraints. The particle positions are modified according to the local flow. Large velocity gradients lead to local accumulation or spreading of particles, which result in a loss of accuracy of the computation. Non-linear stability implies that particle paths are not allowed to cross, which results in the time-step constraint $d t \leq C\|\boldsymbol{\nabla} \boldsymbol{u}\|_{\infty}^{-1}$. This condition is often less demanding than classical CFL conditions. However, it implies that maintaining a regular particle distribution is an important issue. Therefore rather irregular particle positions are projected onto a regular mesh by interpolating the particle data onto the grid. Thereafter new particles are created at regularly distributed positions. Solving the diffusion part of the equations uses explicit solvers that are stable under the condition $\nu d t \leq C h^{-2}$, which is generally not a severe limitation for $3 \mathrm{D}$ computations with moderate to high Reynolds numbers.

To summarize, each time step of the method consists of the following sequence:

- Interpolate particle vorticity onto the grid.

- Compute vector potential, velocity, and strain on the grid using an FFT-based Poisson solver.

- Interpolate velocity and strain at the particle positions.

- Compute updated values of particle vorticity and location.

- Re-mesh particles on the grid.

- Solve viscous part of the equations with a Particle Strength Exchange (PSE) algorithm.

For more details the reader is referred to [4], [7], and [21].

\section{INTERPOLATION FORMULAS}

The computation of velocity and strain and the creation of new particles through the re-gridding process requires frequent transfers between grid and particles. All these operations are performed with the same interpolation kernels. Assume, for instance, that we are given a distribution of particles located at $x_{p}$ carrying circulations $\boldsymbol{\alpha}_{p}$. With an interpolation kernel $W$ the new circulations $\tilde{\boldsymbol{\alpha}}_{i}$ at the grid points $\tilde{\boldsymbol{x}}_{i}$ are computed from the following formula

$$
\tilde{\boldsymbol{\alpha}}_{i}=\sum_{p} \boldsymbol{\alpha}_{p} W\left(\frac{\tilde{\boldsymbol{x}}_{i}-\boldsymbol{x}_{p}}{h}\right)
$$


where $h$ is the grid size. The smoothing length of $W$ is proportional to $h$ in general. As in our case the volumes of the particles are equal to the volumes of the grid cells, eq. (4) is equivalent to

$$
\tilde{\boldsymbol{\omega}}_{i}=\sum_{p} \boldsymbol{\omega}_{p} W\left(\frac{\tilde{\boldsymbol{x}}_{i}-\boldsymbol{x}_{p}}{h}\right) .
$$

To obtain the particle velocities $\boldsymbol{u}_{p}$ from the grid values $\tilde{\boldsymbol{u}}_{i}$, we compute

$$
\boldsymbol{u}_{p}=\sum_{i} \tilde{\boldsymbol{u}}_{i} W\left(\frac{\boldsymbol{x}_{p}-\tilde{\boldsymbol{x}}_{i}}{h}\right)
$$

As the two- or three-dimensional interpolation kernels are obtained by tensorial products of one-dimensional kernels, we now focus, for the sake of simplicity, on explanations about the one-dimensional formulas.

A natural way to control the accuracy of re-gridding formulas is by enforcing the original and re-meshed particles to share the same total circulation, linear momentum, angular momentum, and so on

$$
\begin{aligned}
\sum \tilde{\alpha}_{i} & =\sum \alpha_{p} \\
\sum \tilde{\alpha}_{i}\left(x-\tilde{x}_{i}\right) & =\sum \alpha_{p}\left(x-x_{p}\right) \\
\sum \tilde{\alpha}_{i}\left(x-\tilde{x}_{i}\right)^{2} & =\sum \alpha_{p}\left(x-x_{p}\right)^{2} \\
& \ldots
\end{aligned}
$$

A hierarchy of interpolating kernels with increasing number of preserved moments and increasing order of accuracy can be constructed this way [7]. The three-point interpolation kernel conserving the first three moments is

$$
\Lambda_{2}(x)= \begin{cases}1-x^{2} & \text { if }|x| \leq 0.5 \\ (1-|x|)(2-|x|) / 2 & \text { if } 0.5<|x| \leq 1.5 \\ 0 & \text { if }|x| \geq 1.5\end{cases}
$$

Although successfully used in the past this kernel has a major drawback. It is not continuous and can create or amplify spurious oscillations. There are two possibilities to avoid this problem: increase the number of preserved moments, or increase the regularity of the interpolation function. The following kernel, which uses four points and preserves one additional moment, is continuous

$$
\Lambda_{3}(x)= \begin{cases}\left(1-x^{2}\right)(2-|x|) / 2 & \text { if }|x| \leq 1 \\ (1-|x|)(2-|x|)(3-|x|) / 6 & \text { if } 1<|x| \leq 2 \\ 0 & \text { if }|x| \geq 2\end{cases}
$$

A more regular kernel is $M_{4}$. It uses four points, is $C^{2}$, but preserves only two moments

$$
M_{4}(x)= \begin{cases}(2-|x|)^{3} / 6-4(1-|x|)^{3} / 6 & \text { if }|x| \leq 1 \\ (2-|x|)^{3} / 6 & \text { if } 1<|x| \leq 2 \\ 0 & \text { if }|x| \geq 2\end{cases}
$$

Monaghan [20] proposed the well-known $M_{4}^{\prime}$ from a combination of $M_{4}$ and its first derivative. This kernel requires four grid points, is $C^{1}$ and preserves three moments

$$
M_{4}^{\prime}(x)= \begin{cases}1-5 x^{2} / 2+3|x|^{3} / 2 & \text { if }|x| \leq 1 \\ (2-|x|)^{2}(1-|x|) / 2 & \text { if } 1<|x| \leq 2 \\ 0 & \text { if }|x| \geq 2\end{cases}
$$




\section{Analysis in spectral SPACE}

In the following we briefly summarize the methodology of a modified-differential equation (MDE) analysis in spectral space. For details on the employed algorithm we refer to [14].

We consider the discretization of the Navier-Stokes equation (1a-1c) on a $(2 \pi)^{3}$-periodic domain. Once a numerical scheme is defined, the modified-differential-equation analysis leads to a differential equation for the velocity $\boldsymbol{u}$ in the form of

$$
\frac{\partial \boldsymbol{u}}{\partial t}+\frac{\partial \boldsymbol{F}(\boldsymbol{u})}{\partial \boldsymbol{x}}=\mathcal{G}_{N}
$$

where $\boldsymbol{F}(\boldsymbol{u})$ is the exact flux function and $\mathcal{G}_{N}$ is the truncation error of the discretization. If $\mathcal{G}_{N}$ approximates the divergence of the subgrid stress tensor in some sense for a finite grid size we obtain an implicit subgrid-scale model contained within the discretization. Using Fourier transforms the MDE can be written in spectral space as

$$
\frac{\partial \widehat{\boldsymbol{u}}_{C}}{\partial t}+\mathrm{i} \boldsymbol{P}(\boldsymbol{\xi}) \cdots \widehat{\boldsymbol{u}_{C} \boldsymbol{u}_{C}}+\nu \boldsymbol{\xi}^{2} \widehat{\boldsymbol{u}}_{C}=\widehat{\mathcal{G}}_{C}
$$

The hat denotes the Fourier transform, i is the imaginary unit, and $\boldsymbol{\xi}$ is the wave-number vector. The tensor $\boldsymbol{P}(\boldsymbol{\xi})$ is defined by $P_{l m n}(\boldsymbol{\xi})=\xi_{m} \delta_{l n}-\xi_{l} \xi_{m} \xi_{n}|\boldsymbol{\xi}|^{-2} . \quad \xi_{C}=N / 2$ is the cut-off wave number and $N$ is the corresponding number of grid points in one dimension. A physical-space discretization covers contributions to the numerical solution to wave numbers up to $|\boldsymbol{\xi}|=\sqrt{3} \xi_{C}$. On the represented wave-number range the kinetic energy is

$$
\widehat{E}(\boldsymbol{\xi})=\frac{1}{2} \widehat{\boldsymbol{u}}_{C}(\boldsymbol{\xi}) \cdot \widehat{\boldsymbol{u}}_{C}^{*}(\boldsymbol{\xi})
$$

Multiplying equation (12) by the complex-conjugate $\widehat{\boldsymbol{u}}_{C}^{*}$ of $\widehat{\boldsymbol{u}}_{C}$ we obtain

$$
\frac{\partial \widehat{E}(\boldsymbol{\xi})}{\partial t}-\widehat{T}_{C}(\boldsymbol{\xi})+2 \nu \boldsymbol{\xi}^{2} \widehat{E}(\boldsymbol{\xi})=\widehat{\boldsymbol{u}}_{C}^{*}(\boldsymbol{\xi}) \cdot \widehat{\mathcal{G}}_{C}(\boldsymbol{\xi})
$$

where $\widehat{T}_{C}(\boldsymbol{\xi})$ is the nonlinear energy transfer and $\varepsilon_{v i s}(\boldsymbol{\xi})=2 \nu \boldsymbol{\xi}^{2} \widehat{E}(\boldsymbol{\xi})$ is the molecular dissipation. The righthand side of this equation is the numerical dissipation

$$
\varepsilon_{n u m}(\boldsymbol{\xi})=\widehat{\boldsymbol{u}}_{C}^{*}(\boldsymbol{\xi}) \cdot \widehat{\mathcal{G}}_{C}(\boldsymbol{\xi})
$$

implied by the discretization. Now we investigate how to model the physical subgrid dissipation $\varepsilon_{S G S}$ by $\varepsilon_{n u m}$.

An exact match between $\varepsilon_{n u m}$ and $\varepsilon_{S G S}$ cannot be achieved since $\varepsilon_{S G S}$ involves interactions with nonrepresented scales. For modeling it is therefore necessary to invoke theoretical energy-transfer expressions. Employing an eddy-viscosity hypothesis the subgrid-scale dissipation is

$$
\varepsilon_{S G S}(\boldsymbol{\xi})=2 \nu_{S G S} \xi^{2} \widehat{E}(\boldsymbol{\xi})
$$

Similarly, the numerical dissipation can be expressed as

$$
\nu_{n u m}=\frac{\varepsilon_{n u m}(\boldsymbol{\xi})}{2 \xi^{2} \widehat{E}(\boldsymbol{\xi})} .
$$

In general $\nu_{\text {num }}$ is a function of the wavenumber vector $\boldsymbol{\xi}$. For isotropic turbulence, however, statistical properties of eq. (14) follow from the scalar evolution equation for the 3D energy spectrum

$$
\frac{\partial \widehat{E}(\xi)}{\partial t}-\widehat{T}_{C}(\xi)+2 \nu \xi^{2} \widehat{E}(\xi)=\varepsilon_{n u m}(\xi) \text {. }
$$



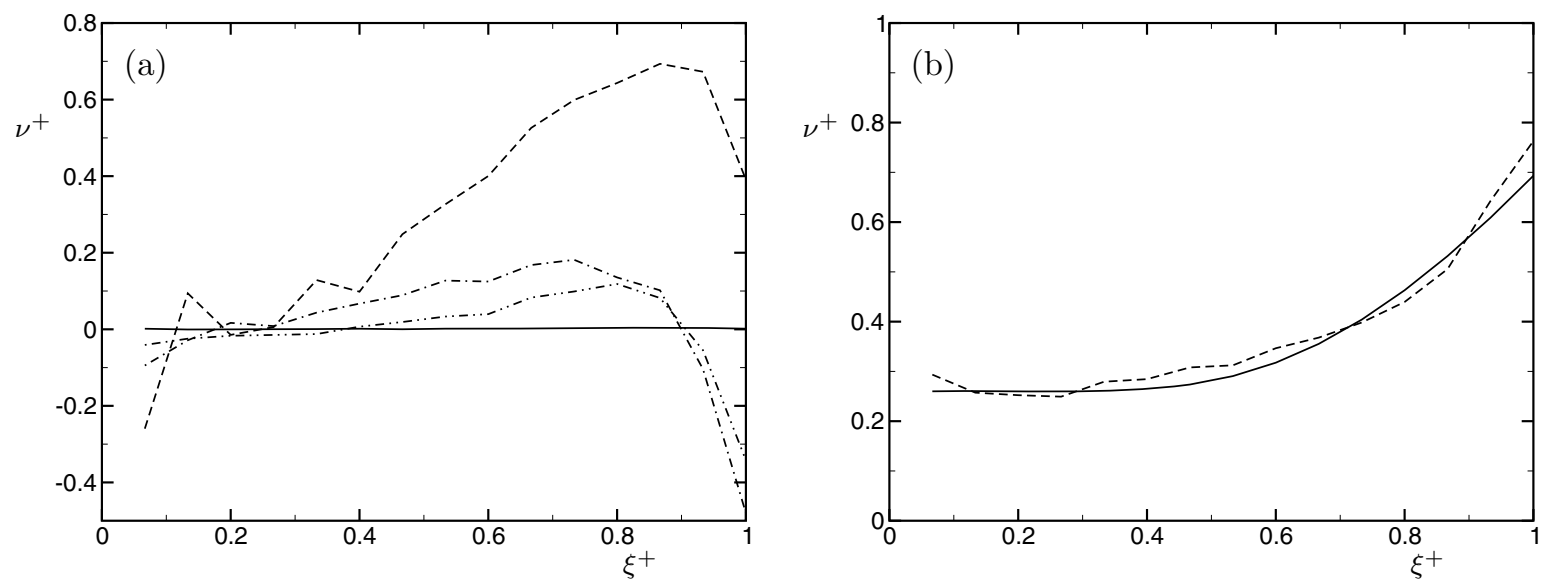

FIGURE 1. Spectral numerical viscosity of (a) - de-aliased spectral method, $\cdot-\cdot-\cdot-2$ ndorder central FD, - - - - - 4th-order central FD, - - - -2 nd-order central FD with staggered grid. (b) - EDQNM theory [3], - - - ALDM with optimized parameters [14].

For a given numerical scheme $\nu_{n u m}(\xi)$ can be computed from

$$
\nu_{n u m}(\xi)=-\frac{1}{2 \xi^{2} \widehat{E}(\xi)} \int_{|\boldsymbol{\xi}|=\xi} \widehat{\boldsymbol{u}}_{C}^{*}(\boldsymbol{\xi}) \cdot \widehat{\mathcal{G}}_{N}(\boldsymbol{\xi}) d \boldsymbol{\xi}
$$

Convenient for our purposes is a normalization by

$$
\nu_{n u m}^{+}\left(\xi^{+}\right)=\nu_{n u m}\left(\xi^{+} \xi_{C}\right) \sqrt{\frac{\xi_{C}}{\widehat{E}\left(\xi_{C}\right)}}, \text { with } \xi^{+}=\frac{\xi}{\xi_{C}}
$$

In the following the objective is to define model parameters and to adjust them in such a way that the implicit model's dissipative properties are consistent with analytical theories of turbulence. The concept of a wavenumber-dependent spectral eddy viscosity was first proposed by Heisenberg [13]. For high Reynolds numbers and under the assumption of a Kolmogorov range Chollet [3] proposes the expression

$$
\nu_{\text {Chollet }}^{+}\left(\xi^{+}\right)=0.441 C_{K}^{-3 / 2}\left(1+34.47 e^{3.03 \xi^{+}}\right)
$$

as best fit to the exact solution, where general shape and the pre-factor $0.441 C_{K}^{-3 / 2}$ is also supported by EDQNM turbulence theory [19].

The spectral numerical viscosity of a discretization scheme is analyzed in the specific real-world environment of a numerical simulations of three-dimensional freely decaying homogeneous isotropic turbulence. For this purpose a trial computation is advanced for a small number of time steps. An a-posteriori analysis of the data allows to identify the spectral eddy viscosity inherent in the discretization [14]. This method can be applied to most numerical schemes. Since the analysis is performed in spectral space, only a de-aliased spectral discretization will yield $\nu_{n u m}^{+}\left(\xi^{+}\right) \equiv 0$. We have analyzed the numerical viscosity of several standard schemes, such as central finite difference methods (FD), see figure 1. The results are consistent with the analysis performed by Ghosal [11]. 

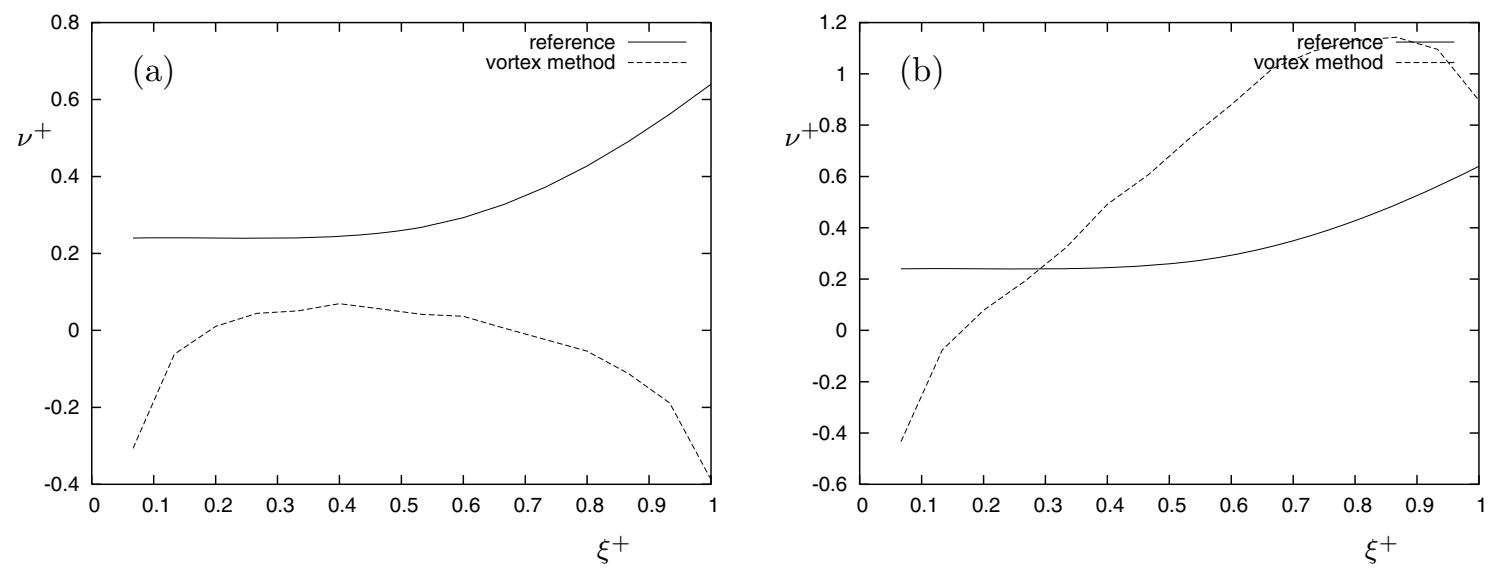

Figure 2. Spectral numerical viscosity of the VIC method with (a) $M_{4}^{\prime}$ and (b) $\Lambda_{3}$.

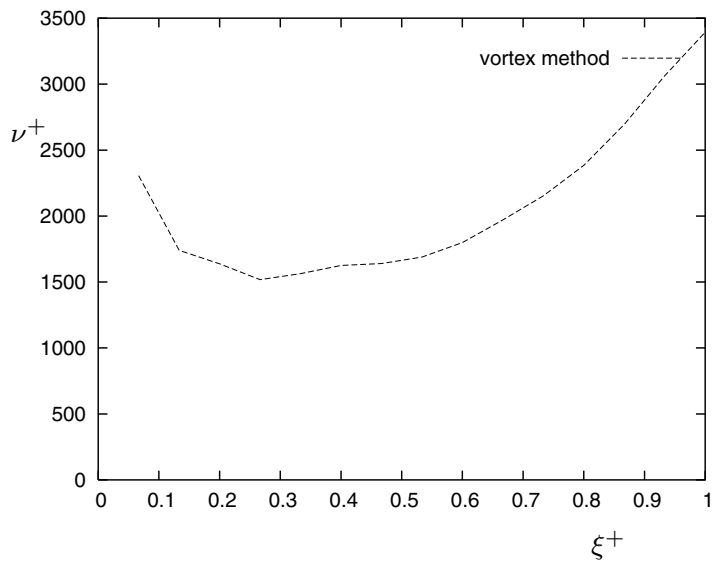

Figure 3. Spectral numerical viscosity of the VIC method with $M_{4}$.

\section{Choice And optimization of Parameters}

Interpolation kernels are a key element of the vortex-in-cell method. In the first part of this section we analyze the spectral numerical viscosity associated with well-established interpolation kernels when used within the VIC method. In the second part, a new kernel for implicit LES is derived from the linear combination of standard kernels. Pursuing this natural approach, the weights are parameters of an implicit sub-grid scale model.

Figure 2 shows the results obtained with $M_{4}^{\prime}$ and $\Lambda_{3}$. The values of the numerical spectral viscosity are acceptable, but the shapes do not match with the theoretical curve. Particularly, the numerical viscosity decreases at the last wave numbers, whereas turbulence theory requires an increase of the eddy viscosity. We also notice that the values of the spectral viscosity are smaller than expected at low wave numbers. However, this ought to be less important for the scheme's ability to model SGS effects.

The magnitude of the numerical viscosity computed for the scheme with kernel $M_{4}$ is huge compared to the theoretical values, see Figure 3. However its global shape is appealing, because the viscosity increases at the last wave numbers. 
At this point, we decide to try to built an implicit SGS model by using a linear combination of the interpolation functions $M_{4}^{\prime}$ and $\Lambda_{3}$, potentially corrected at large wave-numbers by a small contribution of $M_{4}$

$$
K=c_{1} M_{4}^{\prime}+c_{2} \Lambda_{3}+c_{3} M_{4}
$$

Consistency requires $\sum_{i} c_{i}=1$, i.e. two parameters, $c_{2}$ and $c_{3}$, are available for SGS modeling and $c_{1}=1-c_{2}-c_{3}$.
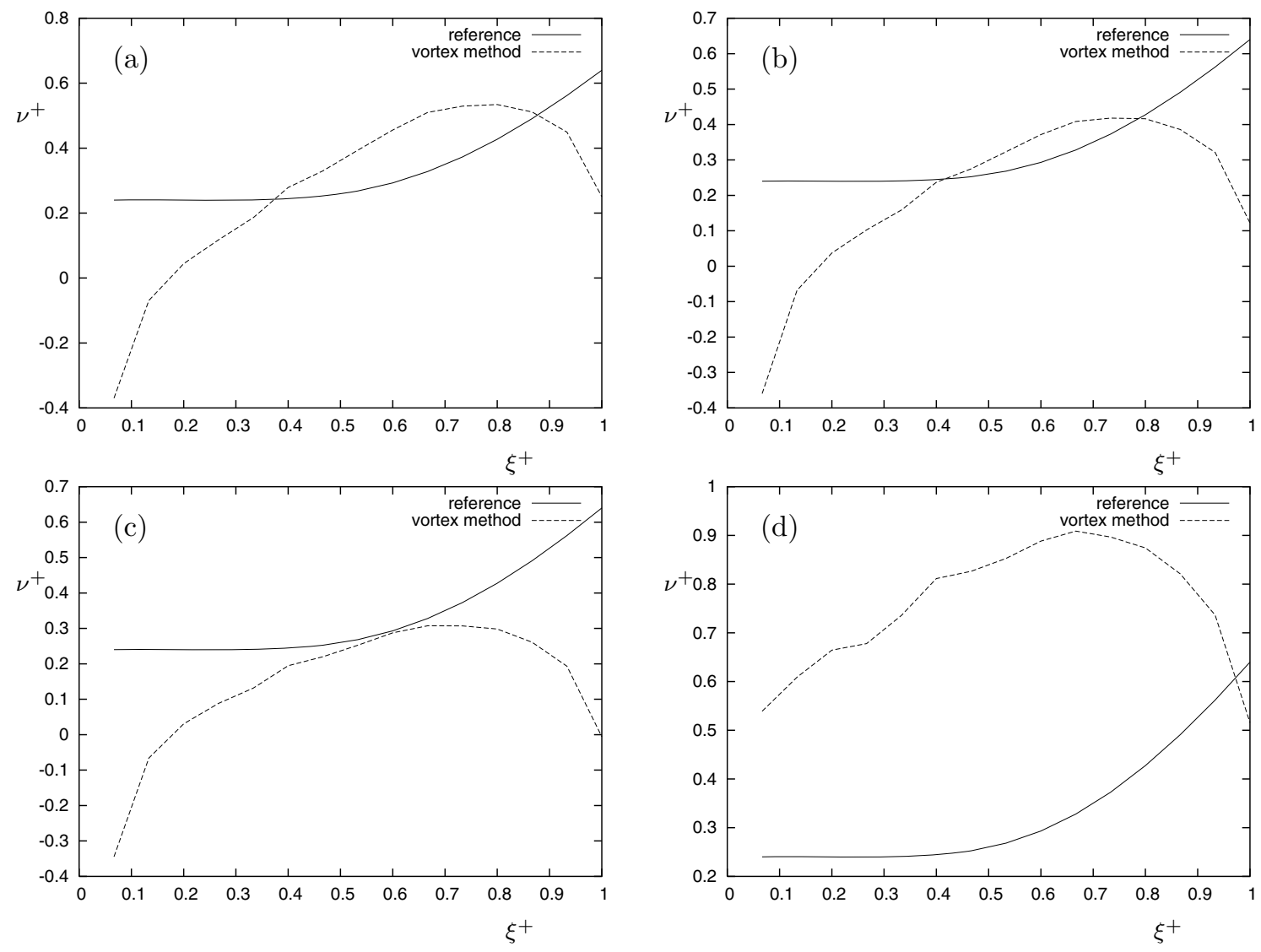

Figure 4. Spectral numerical viscosity of the vortex method with a combination of $M_{4}^{\prime}, \Lambda_{3}$ and $M_{4}$ with respective coefficients (a) $c=(0.5,0.5,0.0)$, (b) $c=(0.6,0.4,0.0)$, (c) $\boldsymbol{c}=(0.7,0.3,0.0)$, and $(\mathrm{d}) \boldsymbol{c}=(0.599,0.4,0.001)$.

Figure 4 shows the numerical spectral viscosity evaluated for several combinations of $M_{4}^{\prime}$ and $\Lambda_{3}$, without $M_{4}$. The result obtained with the combination $c=(0.7,0.3,0.0)$ is not satisfactory, because the numerical viscosity becomes negative at the last wave number and $c=(0.5,0.5,0.0)$ is too dissipative. Hence, the optimum value for $c_{2}$ is bounded by $0.3<c_{2}<0.5$. The combination $c=(0.6,0.4,0.0)$ gives good results in the range of normalized wave-numbers $\xi^{+}=0.3 \ldots 0.8$. We thus decide to consider this as our best combination of $M_{4}^{\prime}$ and $\Lambda_{3}$.

Finally, we study the influence of $M_{4}$ on the spectral viscosity with the combination $\left(0.6-c_{3}, 0.4, c_{3}\right)$. As shown in Figure 4, this additional parameter increases the average value of the spectral numerical viscosity substantially. However, it does not change the shape in the last wave-numbers. This finding reflects the nonlinearity of the Navier-Stokes equations, but it is contrary to what was expected. Consequently, we use the parameters $\boldsymbol{c}=(0.6,0.4,0.0)$ in the following, even if the compliance with EDQNM theory shows room for improvement. 
We do not claim that $(0.6,0.4,0.0)$ is the optimum solution to the given problem. For the purpose of the present study, however, this set of parameters is sufficiently close to the optimum. In order to determine an optimum solution to a nonlinear problem, systematic (and time-consuming) optimization strategies, e.g. an evolutionary optimization algorithm as employed by Adams et al. [1], are required.

\section{Numerical Results}

For an a posteriori validation of the implicit SGS model provided by the VIC method with the selected linear combination of interpolating kernels $M_{4}^{\prime}$ and $\Lambda_{3}$ we perform LES of a 2D Taylor-Green vortex, of a 3D Taylor-Green vortex, and of decaying isotropic turbulence.

All simulations presented in this section are carried out in a $(2 \pi)^{3}$-periodic computational domain, discretized with a uniform cartesian grid of $32 \times 32 \times 32$ cells, and the same number of particles. For time advancement, we use an explicit fourth-order Runge-Kutta scheme.

\subsection{Two-dimensional Taylor-Green vortex}

The two-dimensional Taylor-Green vortex in a periodic domain has an analytical solution that reads

$$
\begin{aligned}
& u_{1}=-\sqrt{a} \cos \left(k x_{1}\right) \sin \left(k x_{2}\right) \exp \left(-(2 k)^{2} t / R e\right) \\
& u_{2}=\sqrt{a} \sin \left(k x_{1}\right) \cos \left(k x_{2}\right) \exp \left(-(2 k)^{2} t / R e\right) \\
& u_{3}=0
\end{aligned}
$$

with $u_{1}, u_{2}, u_{3}$ being the components of the velocity vector. The parameters of our numerical simulation are $k=1, R e=1$, and $a=0.0016$.

The analytical decay exponent of the kinetic energy is $-4 t / R e$. Figure 5 shows the line $y=-4 t$ and the logarithm of the kinetic energy evaluated from the numerical simulation. It is observed that the numerical results are in excellent agreement with the theoretical prediction. The vortex is well-resolved by $32^{3}$ particles.

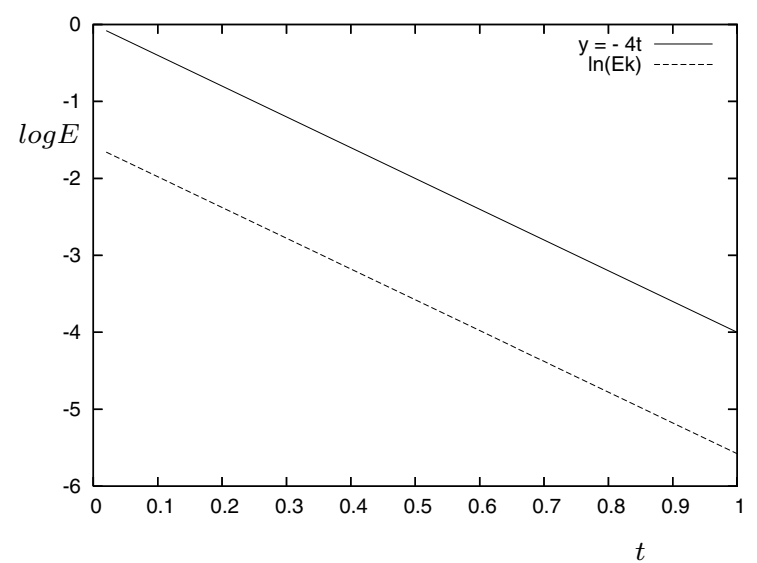

FiguRE 5. Decay of the kinetic energy of the 2D Taylor-Green vortex.

This test validates the correct implementation of the method. 


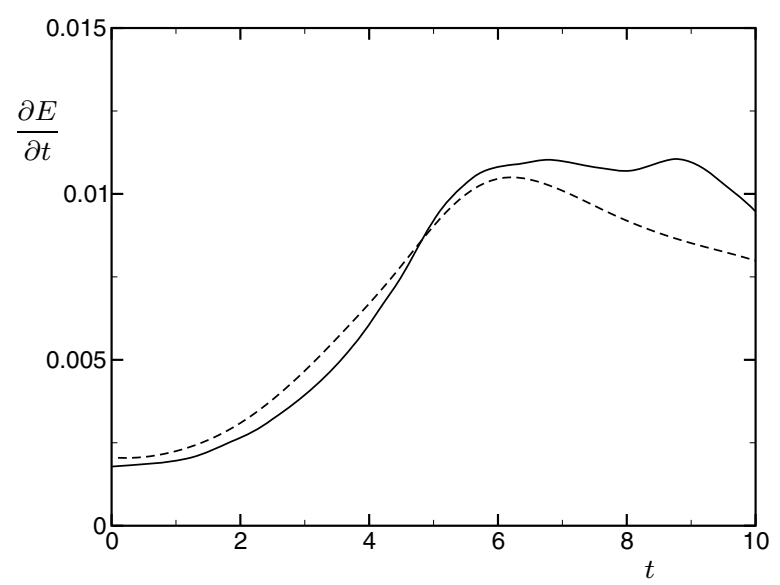

FIGURE 6. Rate of kinetic-energy dissipation for LES of the 3D Taylor-Green vortex at Re $=$ 400. — DNS data from Ref. [2], - - - implicit LES with VIC method.

\subsection{Three-dimensional Taylor-Green vortex}

The 3D viscous Taylor-Green Vortex (TGV) characterized by the initial data

$$
\begin{aligned}
& u_{1}=0 \\
& u_{2}=\cos \left(k x_{1}\right) \sin \left(k x_{2}\right) \cos \left(k x_{3}\right) \\
& u_{3}=-\sin \left(k x_{1}\right) \cos \left(k x_{2}\right) \sin \left(k x_{3}\right)
\end{aligned}
$$

has no analytical solution. It was subject to a large number of investigations since it was supposed that the initially laminar flow develops singularities at $R e \rightarrow \infty$. Numerically, no blow-up has been observed, but there is still no proof [10].

In order to assess the quality of LES using the VIC method, the characteristic growth and decay of the dissipation rate is compared with DNS data. The DNS of Brachet et al. [2] provides detailed reference data for the 3D Taylor-Green vortex. These spectral DNS exploit spatial symmetries of the TGV to reduce the effective computational cost by a factor of 8 . It was therefore possible to resolve Reynolds numbers up to Re $=5000$ on a grid of $864^{3}$ modes. For the present LES, spatial symmetries are not imposed, i.e. the $(2 \pi)^{3}$-periodic computational domain contains 8 counter-rotating vortices and $k=1$ in eq. (24).

Using a grid of $32^{3}$ cells, a fair comparison of LES with Brachet's DNS is possible for Reynolds numbers $\operatorname{Re}=100, \operatorname{Re}=200$, and $\operatorname{Re}=400$. Figure 6 shows the dissipation rate for LES and DNS at Reynolds number Re $=400$. LES and DNS compare reasonably well. Note that the resolution requirement of the DNS is two orders of magnitude higher than that of the LES. These results for the 3D Taylor-Green vortex confirm that the PIC method does not affect the growth of the instability modes of the initially laminar flow and does provide sufficient SGS dissipation to stabilize the simulation during the ensuing quasi-turbulent stage.

\subsection{Comte-Bellot - Corrsin experiment}

A different situation is encountered when the VIC method is applied to decaying grid-generated turbulence. The computations are initialized with energy spectrum and Reynolds numbers adapted to the wind-tunnel experiments of Comte-Bellot and Corrsin [5], denoted hereafter as CBC. Among other space-time correlations CBC provides streamwise energy spectra for grid-generated turbulence at three positions downstream of a mesh. Table 3 of Ref. [5] gives corresponding 3D energy spectra which were obtained under the assumption of isotropy. 

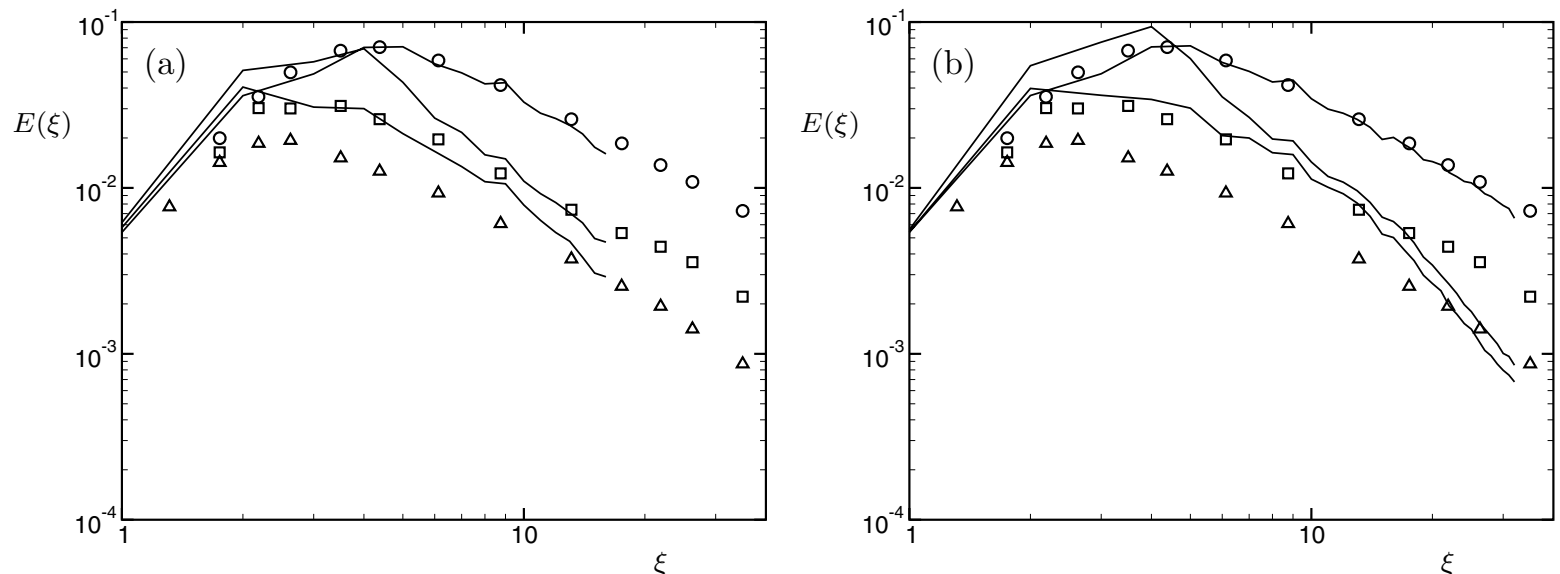

FIGURE 7. Instantaneous 3D energy spectra for LES with the VIC method of the ComteBellot - Corrsin experiment. (a) LES with $32^{3}$ cells/particles, $d t=0.03$. (b) LES with $64^{3}$ cells/particles, $d t=0.015$. Symbols represent experimental data of Ref. [5] $\left(\bigcirc t^{\prime}=42, \square\right.$ $t^{\prime}=98$ and $\triangle t^{\prime}=171$ ), solid lines denote the present LES.

In the simulation this flow is modeled as decaying turbulence in a $(2 \pi)^{3}$-periodic computational domain. Based on the Taylor hypothesis the temporal evolution in the simulation corresponds to a downstream evolution in the wind-tunnel experiment with the experimental mean-flow speed which is approximately constant. The energy distribution of the initial velocity field is matched to the first measured 3D energy spectrum of CBC. The SGS model is verified by comparing computational and experimental 3D energy spectra at later time instants which correspond to the other two measuring stations. For more details, particularly with respect to non-dimensionalization and initial-data generation, we refer to [14].

In this test case a correct representation of the energy containing range of the spectrum is as important as the proper modeling of the subgrid stresses. The present VIC method does not satisfactorily meet these requirements. The large-scale turbulence decays too slowly, whereas the method over-predicts the dissipation at the smallest resolved scales, see fig. 7a. The situation does not change when the number of particles is increased. Figure $7 \mathrm{~b}$ shows results from a LES with $64 \times 64 \times 64$ cells and the same number of particles.

\section{Conclusions}

We have proposed a new approach to design interpolation kernels assigned to the specific use in the implicit large-eddy simulation (LES) of turbulent flows. In implicit LES the subgrid-scale model is implicitly contained within the discretization. An explicit computation of model terms therefore becomes unnecessary.

The numerical truncation error of the vortex-in-cell method is analyzed a-posteriori through the effective spectral numerical viscosity in simulations of three-dimensional isotropic turbulence. The interpolation kernels used for velocity-smoothing and re-meshing were identified as the most relevant components affecting the shape of the spectral numerical viscosity. Turbulence theories, such as EDQNM [3,19], define strict requirements for the spectral eddy viscosity to be met by a SGS model. The inherent eddy viscosity of standard kernels is found to be insufficient.

The dissipative properties of the investigated kernels differ considerably. Exploiting these differences, linear combination of three kernels lead to new kernels with improved numerical viscosity. The present study is intended as proof of this concept. It is shown that the truncation error of the vortex-in-cell method with optimized interpolation kernels can act as implicit SGS model. The present implementation, however, is suitable 
for implicit LES to only a limited extent. Simulations of different Taylor-Green vortices give encouraging results, whereas the predictions for decaying isotropic turbulence shows room for improvement.

The investigated kernels consume no extra computational resources because the linearity of the discretization is preserved. However, this advantage can also be considered as a major drawback. Explicit SGS modeling has clearly demonstrated the benefits of dynamic, solution-adaptive strategies, which correspond to non-linear discretization schemes in implicit LES.

Future interpolation kernels will incorporate more degrees of freedom allowing for an improved agreement of the spectral numerical viscosity with turbulence theory. Our results suggest that a solution-adaptive strategy, e.g. through local time-stepping or nonlinear weight functions, may be required.

\section{REFERENCES}

[1] N.A. Adams, S. Hickel, and S. Franz. Implicit subgrid-scale modeling by adaptive deconvolution. J. Comp. Phys., 200:412-431, 2004.

[2] M.E. Brachet, M. Meneguzzi, A. Vincent, H. Politano, and P.-L. Sulem. Numerical evidence of smooth self-similar dynamics and possibility of subsequent collapse for three-dimensional ideal flow. Phys. Fluids, 4:2845-2854, 1992.

[3] J.-P. Chollet. Two-point closures as a subgrid-scale modeling tool for large-eddy simulations. In F.Durst and B.E. Launder, editors, Turbulent Shear Flows IV, pages 62-72, Heidelberg, 1984. Springer.

[4] J.P. Christiansen. Numerical simulation of hydrodynamics by the method of point vortices. J. Comput. Phys., 13:363-379, 1973.

[5] G. Comte-Bellot and S. Corrsin. Simple Eulerian time correlation of full and narrow-band velocity signals in grid-generated 'isotropic' turbulence. J. Fluid Mech., 48:273-337, 1971.

[6] G.-H. Cottet. Artificial viscosity models for vortex and particle methods. J. Comput. Phys., 127:299-308, 1996.

[7] G.-H. Cottet and P.D. Koumoutsakos. Vortex methods: Theory and Practice. Cambridge University Press, Cambridge, UK, 2000 .

[8] G.-H. Cottet and L. Weynans. Particle methods revisited: a class of high-order finite-difference methods. C. R. Acad. Sci. Paris, Ser. I, submitted( ): , 2006.

[9] J. A. Domaradzki and D. D. Holm. Navier-Stokes-alpha model: LES equations with nonlinear dispersion. In B. Geurts, editor, Modern Simulation Strategies for Turbulent Flow, pages 107-122. R. T. Edwards, 2001.

[10] U. Frisch. Turbulence. Cambridge University Press, 1995.

[11] S. Ghosal. An analysis of numerical errors in large-eddy simulations of turbulence. J. Comput. Phys., 125:187-206, 1996.

[12] F.F. Grinstein, L.G. Margolin, and W.G. Rider, editors. Implicit Large Eddy Simulation: Computing Turbulent Flow Dynamics. Cambridge University Press, Cambridge, UK, 2007.

[13] W. Heisenberg. Zur statistischen Theorie der Turbulenz. Z. Phys. A, 124:628-657, 1948.

[14] S. Hickel, N.A. Adams, and J.A. Domaradzki. An adaptive local deconvolution method for implicit LES. J. Comp. Phys., 213:413-436, 2006.

[15] S. Hickel, T. Kempe, and N.A. Adams. On implicit subgrid-scale modeling in wall-bounded flows. In Proceedings of the EUROMECH Colloquium 469, pages 36-37, Dresden, Germany, 2005.

[16] S. Hickel, T. Kempe, and N.A. Adams. Implicit large-eddy simulation applied to turbulent channel flow with periodic constrictions. Theoret. Comput. Fluid Dynamics, 2006. (submitted).

[17] A. Kravchenko and P. Moin. On the effect of numerical errors in large-eddy simulation of turbulent flows. J. Comp. Phys., 130:310-322, 1997.

[18] A. Leonard. Energy cascade in large eddy simulations of turbulent fluid flows. Adv. Geophys., 18A:237-248, 1974.

[19] M. Lesieur. Turbulence in Fluids. Kluwer Academic Publishers, Dordrecht, The Netherlands, 3 edition, 1997.

[20] J. J. Monagan. Extrapolating B splines for interpolation. J. Comput. Phys., 60:253-262, 1985.

[21] M. Ould-Salihi, G.-H. Cottet, and M. El Hamraoui. Blending finite-difference and vortex methods for incompressible flow computations. SIAM J. Sci. Comp., 22:1655-1674, 2000.

[22] U. Schumann. Subgrid scale model for finite-difference simulations of turbulence in plane channels and annuli. J. Comp. Phys., 18:376-404, 1975. 